\title{
Breast Cancer Detection by Optimal Classification using GWO Algorithm
}

\author{
V. Vinolin \\ Department of Applied Electronics, \\ Regional Centre of Anna University Tirunelveli, Tamil Nadu, India \\ v.vinolin@gmail.com
}

\begin{abstract}
This paper intends to develop a novel breast cancer detection model for classifying the normal, benign or malignant patterns in a mammogram. The diagnosis process is done based on three stages such as pre-processing, feature extraction and classification. Initially, the Discrete Fourier Transform (DFT) is applied in the processing stage. Next, to pre-processing, the Gray Level Co-Occurrence Matrix (GLCM) features of the image are extracted. The GLCM-based features are then classified using Support Vector Machine (SVM) for classifying the mammogram. Further, the weights of the SVM are optimized using the Grey Wolf optimization (GWO) model for improving the classification accuracy. This classification mechanism is used to diagnose the benign and malignant patterns in a mammogram. Moreover, the proposed scheme is evaluated over traditional models such as GA, PSO and FF as well as the outcomes is verified.
\end{abstract}

Keywords: Breast cancer Detection, GLCM; SVM classifier; WaterShed Grey Wolf Algorithm.

\begin{tabular}{ll} 
Nomenclature & \\
\hline Abbreviation & Description \\
\hline GA & Generic Algorithm \\
PSO & Particle Swarm Optimization \\
FF & FireFly Algorithm \\
DuSAT & Dual Stage Adaptive Thresholding \\
ADC & Abnormality Detection Classifier \\
ELM & Extreme Learning Machine \\
SVM & Support Vector Machine \\
NB & Naive Bayesian \\
DFT & Discrete Fourier Transform \\
GLCM & Gray Level Co-Occurrence Matrix \\
SVM & Support Vector Machine \\
\hline
\end{tabular}

\section{Introduction}

Breast cancer [1] [2] [3] remains as one of the major reasons for fatality causes for women. Before the spreading of cancer to the remaining parts of the body, breast cancer should be diagnosed at the initial stage, so that suitable treatment could be made. The screening mammography is considered as a better technique in treating breast cancer in women [4] [5]. Mammography is capable of identifying the clinical difficulties related to benign fibrosis. Mammographic inefficiencies [6] [7] in breast tumour was categorized into two major types namely; masses and calcifications. A wide variety of digital mammography systems are now present. Nevertheless, these vary greatly in terms of physical performance and cost; ranging from expensive direct Digital Mammography Systems (DR) to less expensive Computerized Radiography (CR) systems.

Depending on the analyses conducted in South Australia, the mammography was found to lessen the rate of death by 41\%. Throughout the screening mammography [8] [9] [10], various investigations have revealed that $11-25 \%$ of breast cancers were not detected at the earlier stage. In India, over, 10,000 breast cancer [11] [12] patients are found to be treated annually. In 2009, the Harvard School of Public Health has carried out an analysis on breast cancer [13] [14] and it has revealed that globally, 1.35 million cases of patients require treatment for cancer. Breast cancer [15] [16] cases were predicted to rise (C) Resbee Publishers https://doi.org/10.46253/j.mr.v2i2.a2 
Breast Cancer Detection by Optimal Classification using GWO Algorithm

by $26 \%$ in 2020 and the majority of these would be noticed in developing countries. Radiologists cautiously explore each mammogram [17] [18] in the screening course of action for detecting any visual indications of abnormality. In addition, the visual clues were not clear and will be differing in appearance, thus making analysis challenging in the earlier stage for specialists [19] [20].

The major contribution of the paper is based on three stages namely, pre-processing, feature extraction and classification. During pre-processing, the mammogram image is subjected to DFT for filtering the image. Further, the GLCM-based features are extracted from the pre-processed image. Then the extracted GLCM-based features are classified using SVM classifier, in which the weights are optimized using GWO algorithm. Further, the proposed model is compared with the conventional algorithms and the results are attained.

The arrangement of the work is as follows. Section 2 discusses the reviews done on breast cancer detection. Section 3 describes the framework of breast cancer diagnosis and section 4 analyses the phases in breast cancer diagnosis. Moreover, section 5 portrays the objective model on GWO-based SVM for breast cancer detection. In addition, section 6 explains the results and section 7 concludes the paper.

\section{Literature Review}

\subsection{Related Works}

In 2017, J. Anitha et al. [1] introduced a new DuSAT technique in mammogram detection. At first, the adaptive thresholding was exploited to carry out segmentation and accordingly, the best global threshold was chosen by exploiting the class standard deviation. Finally, an adaptive window based model was performed for obtaining an improved segmentation. Thus the local and global thresholding enhances the accuracy of recognition in an optimal manner.

In 2015, Chun-Chu Jen and Shyr-Shen Yu [2] have developed a novel approach for abnormal mammograms using mammographic datasets. This scheme was dependent on the novel ADC and it was carried out with the least number of gradients, $1^{\text {st }}$ order statistical characteristics, and intensities. Initially, image-pre-processing techniques were examined to attain accurate breast segmentation such as binarization, muscle suppression, and de-noising. Further, for determining the feature weights, the Principle Component Analysis (PCA) method was adopted.

In 2015, Al-Najdawia et al. [3] introduced mammogram development and segmentation techniques. The development of the presented model was carried out on mammogram images, in order to improve their performance and thus the existing noise could be reduced. In addition, it helps the radiologists in the recognition of errors. Further, various combinations of conventional, noise reduction, and featurebased contrast improvement techniques were analyzed. Thus the implemented model was found to improve the visual information, thus aiding in the process of segmentation.

In 2015, Xie [4] presented a CAD technique for the analysis of breast cancer depending on the ELM. In the pre-processing stages, it eradicates the interference in a mammographic image. Consequently, the level set form was adopted to divide the pre-processed image. Accordingly, the feature selection was made using the SVM and ELM. Moreover, for differentiating malignant masses from benign ones, a most favorable subset of feature vectors was given as input into the classifiers.

In 2015, Karabatak [5] suggested a novel weighted NB classifier for breast cancer recognition. Here, to prevail over the disadvantages of the presented classifier, a weighted NB classifier was suggested. Moreover, to estimate the presentation of the proposed system, a variety of experiments was carried out and accordingly, the research was examined using 5-fold analysis. Furthermore, varied performance assessment schemes such as accuracy specificity and sensitivity were measured.

\section{Framework of Breast Cancer Detection}

\subsection{Proposed Architecture}

Fig. 1 shows the pictorial representation of the presented model. Let the input mammogram image be indicated by $I$, which is subjected to pre-processing by means of DFT model. The resultant output from the pre-processed image is subjected to feature extraction, where the GLCM features are extracted. GLCM includes four directions, such as $0^{\circ}, 90^{\circ}, 180^{\circ}$, and $270^{\circ}$. These extracted GLCM features are given to SVM classifier, where the weights are optimized by means of the GWO algorithm. Also, it determines the nature of breast cancer, whether it is normal, benign or malignant. Thus the classified breast cancer image can be obtained with improved classification accuracy. 
Breast Cancer Detection by Optimal Classification using GWO Algorithm

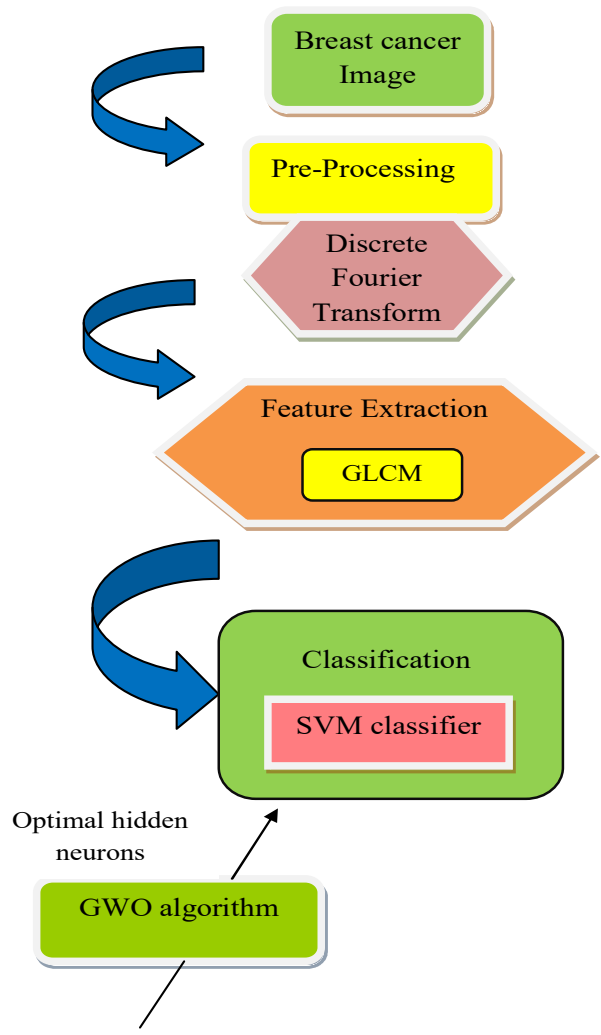

Fig. 1. Diagrammatic representation of the proposed model

\section{Phases in Breast Cancer Diagnosis}

\subsection{Pre-Processing}

The image I taken for diagnosing breast cancer is pre-processed by means of DFT model.

DFT [24]: It is a method that permits us to configure numerically stable and simple criteria for the convergence of general type. Assume that $\Psi_{n}=e^{-\frac{2 \pi j}{n}}$ be a $n^{\text {th }}$ root of unity. Subsequently, in an $n-$

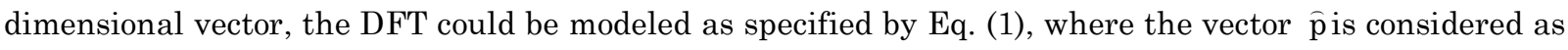
the discrete frequency spectrum of $p$. The inverse transformation is exposed by Eq. (2) and it is easier to derive. The difference operator $\Delta$ is considered for approximating the discrete differentiation. The difference spectrum in the frequency domain is given by Eq. (3)

$$
\begin{aligned}
& \hat{\mathrm{p}}_{\mathrm{i}}=\sum_{\mathrm{k}=0}^{\mathrm{n}} \Psi_{\mathrm{n}}^{\mathrm{ik}} \mathrm{p}_{\mathrm{k}} \\
& \mathrm{p}_{\mathrm{i}}=\frac{1}{\mathrm{n}} \sum_{\mathrm{k}=0}^{\mathrm{n}-1} \Psi_{\mathrm{n}}^{-\mathrm{ik}} \widehat{\mathrm{p}}_{\mathrm{k}} \\
& \Delta^{\mathrm{k}} \hat{\mathrm{p}}:=\left[\left(\Psi_{\mathrm{n}}^{-\mathrm{i}}-1\right)^{\mathrm{k}} \hat{\mathrm{p}}_{\mathrm{i}}\right]_{\mathrm{i}=0}^{\mathrm{n}-1}
\end{aligned}
$$

Thus the filtered breast cancer image using DFT is denoted by $\mathrm{I}_{\mathrm{DFT}}$.

\subsection{Feature Extraction}

The pre-processed image $\mathrm{I}_{\mathrm{DFT}}$ is subjected to feature extraction that extracts the GLCM [23] features. The GLCM features are shown in Table 1. 
Breast Cancer Detection by Optimal Classification using GWO Algorithm

\begin{tabular}{|c|c|}
\hline Entropy & Ent $=-\sum_{\mathrm{i}} \sum_{\mathrm{j}} \mathrm{g}_{\mathrm{ij}} \log _{2} \mathrm{~g}_{\mathrm{ij}}$, where $\mathrm{g}_{\mathrm{ij}}$ denotes the $(\mathrm{i}, \mathrm{j})^{\mathrm{th}}$ entry in GLCM \\
\hline Energy & $\mathrm{E}=\sum_{\mathrm{i}} \sum_{\mathrm{j}} \mathrm{g}_{\mathrm{ij}}^{2}$ \\
\hline Variance & $V=\sum_{i} \sum_{j}(i-\mu)^{2} g_{i j}$, where $\mu$ is the mean of $g_{i j}$ \\
\hline Contrast & Con $=\sum_{i} \sum_{j}(i-j)^{2} g_{i j}$ \\
\hline Correlation & $\begin{array}{l}\mathrm{C}=\frac{\sum_{\mathrm{i}} \sum_{\mathrm{j}}(\mathrm{ij}) \mathrm{g}_{\mathrm{ij}}-\mu_{\mathrm{x}} \mu_{\mathrm{y}}}{\sigma_{\mathrm{x}} \sigma_{\mathrm{y}}}, \text { where } \mu_{\mathrm{x},} \mu_{\mathrm{y}}, \sigma_{\mathrm{x}}, \sigma_{\mathrm{y}} \text { are the mean and standard } \\
\text { deviations of } \mathrm{g}_{\mathrm{x}}, \mathrm{g}_{\mathrm{y}}\end{array}$ \\
\hline Homogeneity & $\mathrm{H}=\sum_{\mathrm{i}} \sum_{\mathrm{j}} \frac{1}{1+(\mathrm{i}-\mathrm{j})^{2}} \mathrm{~g}_{\mathrm{ij}}$ \\
\hline Sum Average (SA) & $\mathrm{SA}=\sum_{\mathrm{i}=2}^{2 \mathrm{~N}_{\mathrm{g}}} \operatorname{ig}_{\mathrm{x}+\mathrm{y}}(\mathrm{i})$, where $\mathrm{N}_{\mathrm{g}}$ denotes the distinct gray levels in the image \\
\hline Sum variance & $A)^{2} g_{x+y}(i)$ \\
\hline Sum entropy & $\mathrm{SE}=\sum_{\mathrm{i}=2}^{2 \mathrm{~N}_{\mathrm{g}}} \mathrm{g}_{\mathrm{x}+\mathrm{y}}(\mathrm{i}) \log \left\{\mathrm{g}_{\mathrm{x}+\mathrm{y}}(\mathrm{i})\right\}$ \\
\hline Difference entropy & $\mathrm{DE}=\sum_{\mathrm{i}=0}^{\mathrm{N}_{\mathrm{g}-1}} \mathrm{~g}_{\mathrm{x}-\mathrm{y}}(\mathrm{i}) \log \left\{\mathrm{g}_{\mathrm{x}-\mathrm{y}}(\mathrm{i})\right\}$ \\
\hline Difference variance & $\mathrm{DV}=$ variance of $\mathrm{g}_{\mathrm{x}-\mathrm{y}}$ \\
\hline $\begin{array}{l}\text { Maximum correlation } \\
\text { coefficient }\end{array}$ & $\left(2^{\text {nd }} \text { largest Eigen value of } Q\right)^{0.5,}$ where $\mathrm{MCC}=\sum_{\mathrm{k}} \frac{\mathrm{g}(\mathrm{i}, \mathrm{k}) \mathrm{g}(\mathrm{j}, \mathrm{k})}{\mathrm{g}_{\mathrm{x}}(\mathrm{i}) \mathrm{g}_{\mathrm{y}}(\mathrm{k})}$ \\
\hline $\begin{array}{l}\text { Information measures of } \\
\text { correlation (IMC1) }\end{array}$ & $\mathrm{IMC1}=\frac{\mathrm{HXY}-\mathrm{HXY} 1}{\max \{\mathrm{HX}, \mathrm{HY}\}}$ \\
\hline $\begin{array}{l}\text { Information measures of } \\
\text { correlation (IMC2) }\end{array}$ & $\begin{array}{l}\text { IMC2 }=\sqrt{(1-\exp [-2.0[H X Y 2-H X Y]])}, \text { where HXY }=-\sum_{i} \sum_{j} g_{i j} \log _{2} g_{i j} \\
\text { HXY1 }=-\sum_{i} \sum_{j} g_{i j} \log _{2}\left\{g_{x}(i) g_{y}(j)\right\} \\
\text { HXY2 }=-\sum_{i} \sum_{j} g_{x}(i) g_{y}(j) \log _{2}\left\{g_{x}(i) g_{y}(j)\right\}\end{array}$ \\
\hline
\end{tabular}

The extracted GLCM-based features are indicated by $\mathrm{F}^{*}$

\subsection{Classification using SVM}

SVM [25] is known as two-class classifier, which produces a hyperplane for classifying two segments of data. The hyperplane of the linear separable problems in an n-dimensional feature space is specified by Eq. (4), which VT indicates the normal vector (weight) and $g$ denotes the distance from hyperplane to origin.

$$
\mathrm{K}(\mathrm{o})=\mathrm{VT}^{\mathrm{T}} \mathrm{C}+\mathrm{q}=0
$$

$\mathrm{K}(\mathrm{o})$ is learned by training data set, $\left\{\mathrm{o}_{\mathrm{i}}, \mathrm{c}_{\mathrm{i}}\right\} ; \mathrm{i}=1, \ldots . \mathrm{h}$, in which $\mathrm{o}_{\mathrm{i}} \in \mathfrak{R}^{\mathrm{n}}$ and $\mathrm{c}_{\mathrm{i}} \in\{+1,1\}$. The training samples are classified precisely by $\mathrm{K}(\mathrm{o})$ with the specified parameters: if $\mathrm{c}_{\mathrm{i}}=+1, \mathrm{~K}(\mathrm{o}) \geq 1$ and if $c_{i}=-1, K(o) \geq-1$. The point that forms $K(x)=+1$ or -1 is known as support vector. Eq. (5) portrays the distance of perpendicular from a particular point a to hyperplane. 
Breast Cancer Detection by Optimal Classification using GWO Algorithm

$$
\mathrm{r}=\frac{\mathrm{VT}^{\mathrm{T}} \mathrm{C}_{\mathrm{n}}+\mathrm{q}}{\|\mathrm{VT}\|}=\frac{\mathrm{y}_{\mathrm{n}}\left(\mathrm{VT}^{\mathrm{T}} \mathrm{C}_{\mathrm{n}}+\mathrm{q}\right)}{\|\mathrm{VT}\|}
$$

The major objective of SVM is the determination of hyperplane to increase the distance among the hyperplane and the training data points, which are nearer to the hyperplane. The corresponding issue is further converted to an equivalent convex quadratic issue as specified by Eq. (6).

$$
\min _{\mathrm{EV}, \mathrm{g}} \frac{1}{2} \| \mathrm{VT}^{2}
$$

So that $c_{i}\left(V^{T}{ }^{\mathrm{T}} \mathrm{o}_{\mathrm{i}}+\mathrm{q}\right)^{3} 1, \mathrm{i}=1,2,3 \ldots \ldots \mathrm{N}$. Using the Lagrange multipliers, Eq. (6) is defined as in Eq. (7).

$$
\begin{aligned}
& \underset{h}{\max } \underset{\mathrm{i}=1}{\mathrm{~N}} \mathrm{~h}_{\mathrm{i}}-\frac{1}{2} \underset{\mathrm{i}=1 \mathrm{j}=1}{\mathrm{~h}} \underset{\mathrm{h}}{\mathrm{h}} \mathrm{c}_{\mathrm{i}} \times \mathrm{c}_{\mathrm{j}} \times \mathrm{h}_{\mathrm{i}} \times \mathrm{h}_{\mathrm{j}} \times\left\langle\mathrm{o}_{\mathrm{i}}, \mathrm{o}_{\mathrm{j}}\right\rangle \mathrm{c} \\
& \text { so that }{\stackrel{\mathrm{h}=1}{\mathrm{~N}} \mathrm{~h}_{\mathrm{i}} \mathrm{c}_{\mathrm{i}}=0}^{\mathrm{N}}=0
\end{aligned}
$$

where $h_{i}, i=1,2 \ldots . N$. The major issue is denoted by $\mathrm{VT}=\sum_{\mathrm{i}=1}^{\mathrm{N}} \kappa_{\mathrm{i}} \mathrm{c}_{\mathrm{i}} \mathrm{o}_{\mathrm{i}}$ and $0=\sum_{\mathrm{i}=1}^{\mathrm{N}} \kappa_{\mathrm{i}} \mathrm{c}_{\mathrm{i}}$. Thus, having the observed Lagrange multipliers $\kappa$, the definition of $w$ and $g$ occurs. In general, data could be overlapped, and thus obtaining of precise training data division is a challenging aspect, and that could lead to least generalization.

\section{Objective model on GWO-based SVM for Breast Cancer Detection}

\subsection{Solution Encoding and Objective Function}

The weights of SVM, which have to be chosen optimally, is given as a solution for encoding as revealed by Fig. 2, where $\mathrm{VT}_{\mathrm{N}}$ denotes the number of weights in SVM.

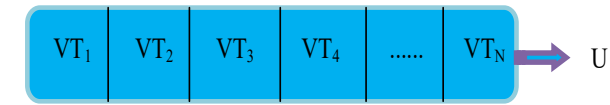

Fig. 2. Solution encoding of the proposed GWO method

\subsection{Proposed Grey Wolf Optimization}

For optimizing the weights, GWO algorithm is adopted in the presented work. The mechanism of GWO [21] algorithm describes the grey wolves' hunting character and its headship hierarchy. There exist 4 types of grey wolves, such as $\alpha, \eta, \zeta, \omega$ that are deployed for performing the leadership nature. Penetrating, circling, and searching the food are the three foremost performances involved in hunting that develop the optimization process.

The wolves $\alpha, \eta$ and $\zeta$ are the major wolves, which focuses on the process of hunting. Among these wolves, $\alpha$ is considered as the leader that makes decisions relating to the hunting process, sleeping location, time to awake, etc. While, $n$ and $\zeta$ holds a $2^{\text {nd }}$ and $3^{\text {rd }}$ level that aids $\alpha$ in taking decisions. In addition, the final level of wolves is concerned as $\omega$, which concern on eating. The encircling characteristics are modeled as per Eq. (10) and Eq. (11), where $X$ and $Y$ denotes coefficient vectors, $\mathrm{U}_{\mathrm{v}}$ indicates prey's position vectors, $v$ denotes position vectors of grey wolves and $t$ specifies present iteration. Eq. (12) and Eq. (13) denotes the model for X and Y, where $a$ is a parameter which is reduced gradually from 2 to 0 in whole iterations. The benchmark formulation of $a$ is given by Eq. (9) and $r_{1}$ and $r_{2}$ specifies the random vectors that lie among $[0,1]$ and $t^{\max }$ denotes the maximum iteration.

$$
\begin{aligned}
& \mathrm{a}_{1}=2-1 *\left(\frac{2}{\mathrm{t}^{\max }}\right) \\
& \mathrm{Z}=\left|\mathrm{Y} \cdot \mathrm{U}_{\mathrm{v}}^{\mathrm{t}}-\mathrm{U}^{\mathrm{t}}\right| \\
& \mathrm{U}_{\mathrm{v}}^{(\mathrm{t}+1)}=\mathrm{U}_{\mathrm{v}}^{\mathrm{t}}-\mathrm{X} \cdot \mathrm{Z} \\
& \mathrm{X}=2 \mathrm{a} \cdot \mathrm{r}_{1}-\mathrm{a} \\
& \mathrm{Y}=2 \mathrm{r}_{2}
\end{aligned}
$$


Breast Cancer Detection by Optimal Classification using GWO Algorithm

The arithmetical formula for describing the hunting character of wolves is given from Eq. (14) to Eq. (19), where the last updated position is specified in Eq. (20), which provides the modified U. The pseudocode of conventional GWO model is specified by algorithm 1.

$$
\begin{aligned}
& \mathrm{Z}_{\alpha}=\left|\mathrm{Y}_{1}-\mathrm{U}_{\alpha}-\mathrm{U}\right| \\
& \mathrm{Z}_{\eta}=\left|\mathrm{Y}_{2}-\mathrm{U}_{\eta}-\mathrm{U}\right| \\
& \mathrm{Z}_{\zeta}=\left|\mathrm{Y}_{3}-\mathrm{U}_{\zeta}-\mathrm{U}\right| \\
& \mathrm{U}_{1}=\mathrm{U}_{\alpha}-\mathrm{X}_{1} \cdot\left(\mathrm{Z}_{\alpha}\right) \\
& \mathrm{U}_{2}=\mathrm{U}_{\eta}-\mathrm{X}_{2} \cdot\left(\mathrm{Z}_{\eta}\right) \\
& \mathrm{U}_{3}=\mathrm{U}_{\zeta}-\mathrm{X}_{3} \cdot\left(\mathrm{Z}_{\zeta}\right) \\
& \mathrm{U}_{\mathrm{v}}^{\mathrm{t}+1}=\frac{\mathrm{U}_{1}+\mathrm{U}_{2}+\mathrm{U}_{3}}{3}
\end{aligned}
$$

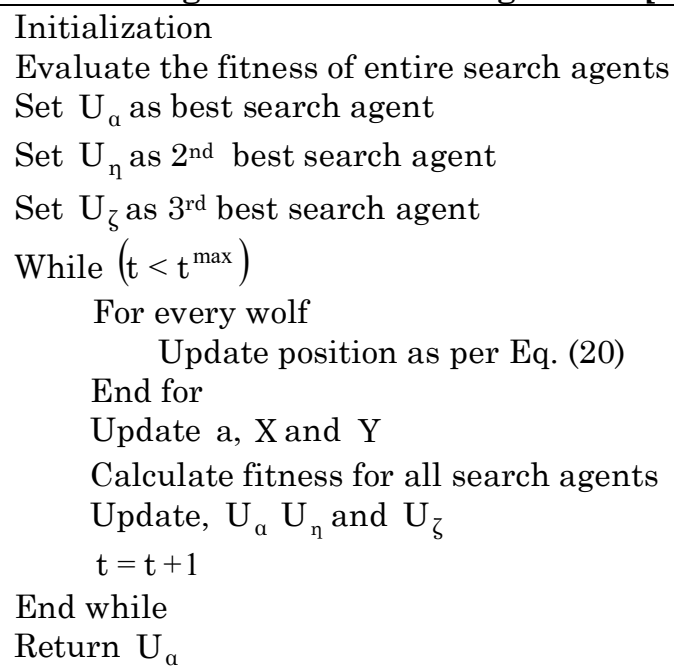

\section{Results and Discussion}

\subsection{Simulation Procedure}

The proposed breast cancer diagnosis model using GWO was simulated using MATLAB and the corresponding results were obtained. In addition, the proposed GWO model was compared with GA [26], PSO [27] and FF [28] and the results were obtained. The experimentation was done based on the performance analysis and overall outcomes were validated. Here, two databases such as MIAS database and the others are extracted from DDSM database.

\subsection{Performance Analysis}

The performance analysis for breast cancer diagnosis for the suggested GWO method is given by Fig. 3 for three measures namely, accuracy, sensitivity, and specificity with respect to the percentage of learning. From Fig. 3(a), the accurateness of the suggested model is found to be enhanced than the other conventional scheme namely GA, PSO, and FF algorithms. Similarly, from Fig. 3(b), the sensitivity of the proposed method has offered improved outcomes with an increase in the percentage of learning. Fig. 3(c) shows the enhanced performance of the implemented model in terms of specificity. Thus the performance analysis of the implemented scheme has been confirmed by means of the experimental analysis. 
Breast Cancer Detection by Optimal Classification using GWO Algorithm

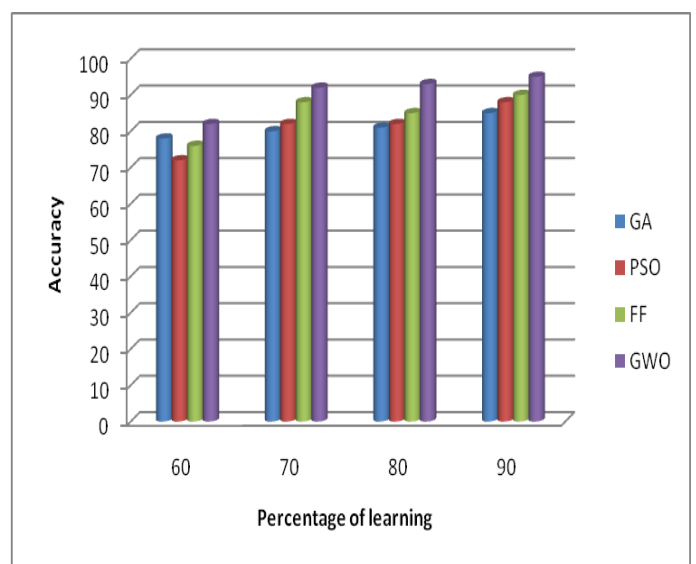

(a)

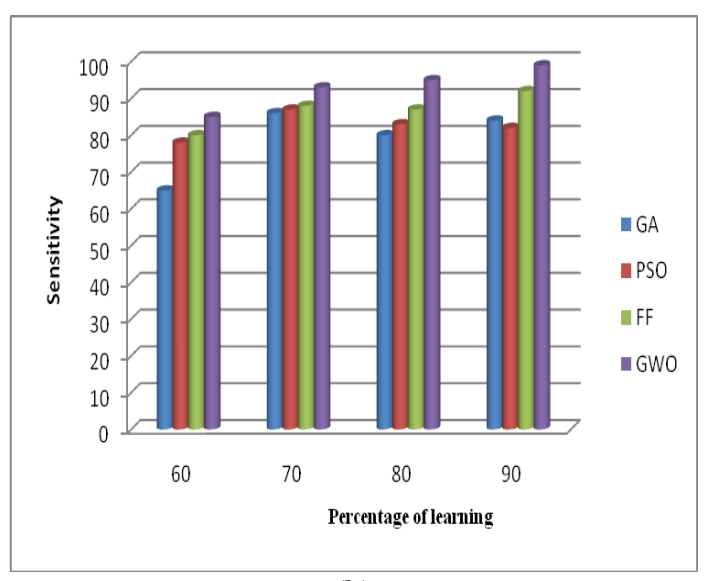

(b)

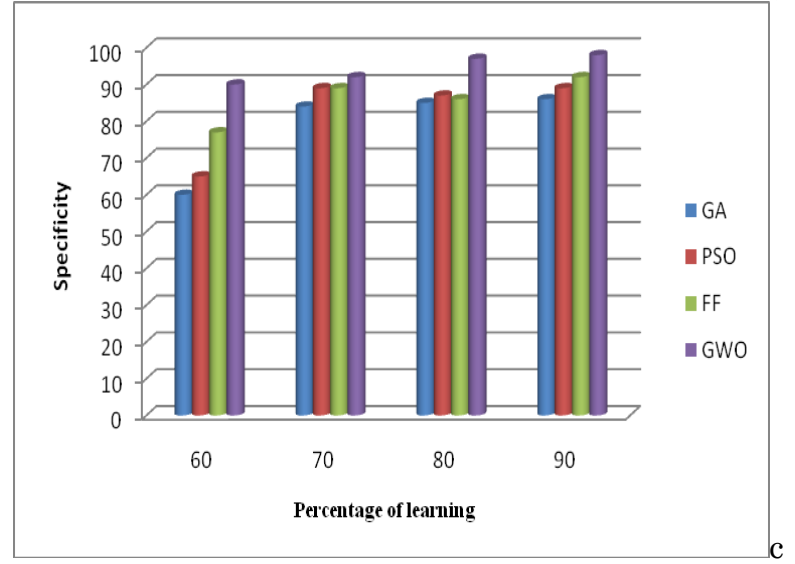

(c)

Fig. 3. Performance analysis of proposed and existing methods in terms of (a) Accuracy (b) Sensitivity (c) Specificity

\subsection{Overall Performance Analysis}

The overall performance for breast cancer diagnosis using the implemented model is specified by Table. 2. From the analysis, better outcomes are offered by the presented GWO model when evaluated over the other traditional models. It can be noted that the adopted model on considering accuracy is $21.4 \%$ better than GA, $11.9 \%$ better than PSO, and $34.93 \%$ better than FF techniques. Likewise, the proposed idea regarding sensitivity is $50.78 \%, 23.31 \%$ and $81.93 \%$ superior to GA, PSO and FF methods. Thus the overall performance analysis of the proposed technique was confirmed from the simulation results.

Table 2: Overall Performance Analysis For The Proposed And Existing Schemes

\begin{tabular}{lcccc}
\hline \multicolumn{1}{c}{ Measures } & GA [26] & PSO [27] & FF [28] & GWO \\
\hline Accuracy & 0.7508 & 0.8421 & 0.880 & 0.954386 \\
Sensitivity & 0.4315 & 0.673 & 0.7894 & 0.873684 \\
Specificity & 0.9105 & 0.9263 & 0.9263 & 0.994737 \\
Precision & 0.7068 & 0.820 & 0.8426 & 0.988095 \\
FPR & 0.0894 & 0.0736 & 0.0736 & 0.005263 \\
FNR & 0.5684 & 0.3263 & 0.210 & 0.126316 \\
NPV & 0.9105 & 0.9263 & 0.9263 & 0.994737 \\
FDR & 0.2931 & 0.1794 & 0.157 & 0.011905 \\
F1-score & 0.5359 & 0.739 & 0.815 & 0.927374 \\
MCC & 0.400 & 0.6343 & 0.728 & 0.897907 \\
\hline
\end{tabular}

\section{Conclusion}

This paper has presented an enhanced breast cancer detection model using GWO algorithm. The diagnosis process was made depending on three stages namely pre-processing, feature extraction and classification. Initially, the image was pre-processed using DFT model. From the pre-processed image, 
Breast Cancer Detection by Optimal Classification using GWO Algorithm

GLCM-based features were extracted. The extracted GLCM features were then classified using the SVM framework, where the weights were optimized using the GWO model. Moreover, the proposed GWO scheme was evaluated over existing scheme s namely, GA, PSO and FF and the results were obtained. From the analysis, the proposed method on considering accuracy was $21.4 \%$ better than GA, $11.9 \%$ better than PSO, and 34.93\% better than FF techniques. Likewise, the proposed GWO scheme in terms of sensitivity was $50.78 \%, 23.31 \%$ and $81.93 \%$ superior to GA, PSO and FF methods. Thus the capability of the adopted GWO algorithm is verified in terms of its performance.

\section{Compliance with Ethical Standards}

Conflicts of interest: Authors declared that they have no conflict of interest.

Human participants: The conducted research follows the ethical standards and the authors ensured that they have not conducted any studies with human participants or animals.

\section{References}

[1] J. Anitha, J. Dinesh Peter, S. Immanuel Alex Pandian, "A dual stage adaptive thresholding (DuSAT) for automatic mass detection in mammograms", Computer Methods and Programs in Biomedicine, vol. 138, pp. 93104, January 2017.

[2] Chun-Chu Jen, Shyr-Shen Yu, "Automatic detection of abnormal mammograms in mammographic images", Expert Systems with Applications, Volume 42, Issue 6, 15 April 2015, Pages 3048-3055.

[3] Nijad Al-Najdawi, Mariam Biltawi, Sara Tedmori, "Mammogram image visual enhancement, mass segmentation and classification", Applied Soft Computing, vol.35, pp.175-185, October 2015.

[4] WeiyingXie, Yunsong Li, Yide Ma,"Breast mass classification in digital mammography based on extreme learning machine",Neurocomputing, vol. 173, Part 3, pp.930-941, 15 January 2016.

[5] Murat Karabatak, "A new classifier for breast cancer detection based on Naïve Bayesian", Measurement, vol. 72 , pp. 32-36, August 2015.

[6] Zhen Yang, Min Dong, YananGuo, Xiaoli Gao, Yide Ma, "A new method of micro-calcifications detection indigitized mammograms based on improved simplified PCNN", Neurocomputing, vol.218, pp. 79-90, 19 December 2016.

[7] Meenakshi M. Pawar, Sanjay N. Talbar, "Genetic Fuzzy System (GFS) based wavelet co-occurrence feature selection in mammogram classification for breast cancer diagnosis", Perspectives in Science, vol. 8, pp.247-250, September 2016.

[8] M. Sameti, R. K. Ward, J. Morgan-Parkes and B. Palcic, "Image Feature Extraction in the Last Screening Mammograms Prior to Detection of Breast Cancer," IEEE Journal of Selected Topics in Signal Processing, vol. 3, no. 1, pp. 46-52, Feb. 2009.

[9] Nandakumar A, Anantha N, Venugopal TC, "Survivalin breast cancer: a population-based study in Bangalore", India. Int J Cancer, 60, 593-6,1995.

[10] Roder, D., Houssami, N., Farshid, G., Gill, G., Luke Downey, P,"Population screening and intensity of screening are associated with reduced breast cancer mortality", Evidence of efficacy of mammography screening in Australia. Breast Cancer Research and Treatment, vol.108(3), pp.409-416, 2008.

[11] Goergen, S., Evans, J., Cohen, G., \& Macmillan, J," Characteristics of breast carcinomas missed by screening radiologists", Radiology, vol.204(11), pp.131-135, 1995.

[12] C.H. Lee, D.D. Dershaw, D. Kopans, P. Evans, B. Monsees, D. Monticciolo, R. J. Brenner, L. Bassett, W. Berg, and S. Feig, "Breast cancer screening with imaging: recommendations from the society of breast imaging and the ACR on the use of mammography, breast MRI, breast ultrasound, and other technologies for the detection of clinically occult breast cancer", J. Amer. Coll. Radiol. vol.7 (1), pp.18-27, 2010.

[13] M. Bajger, F. Ma, M.J. Bottema, "Automatic tuning of MST segmentation of mam-mograms for registration and mass detection algorithms", Digit. Image Comput.pp.400-407,2009.

[14] Sami Dhahbi, WalidBarhoumi, EzzeddineZagrouba, "Breast cancer diagnosis in digitized mammograms using curvelet moments", Computers in Biology and Medicine, vol.64, pp.79-90,2015.

[15] N.C. Tsai,H.W.Chen,S.L.Hsu,"Computer-aided diagnosis forearly-stage breast cancer by using wavelet transform,computerized medical imaging and graphics, Off.J.Comput.Med.ImagingSoc.35,pp.1-8,2011.

[16] R.Lakshmanan,V.Thomas,"Enhancement of micro calcification features using morphology and contourlet transform",Proceedings of the International Conference onAdvances in Computing and Communications,pp.1417,2012 .

[17] S.-N. Yu,K.-Y.Li,Y.-K.Huang,"Detection of microcalcifications in digital mammograms using wavelet filter and Markov random field model",Comput.Med. ImagingGraph.30,163-173, 2001.

[18] S. Yu,L.Guan, "ACAD system for the automatic detection of clustered micro-calcifications in digitized mammogram films", IEEETrans.Med.Imaging19,pp. 115-126,2000.

[19] H. Soltanian-Zadeh,F.Rafiee-Rad, "Comparison of multiwavelet, wavelet,Haralick, and shape features for microcalcification classification in mammograms","Pattern Recognit.37,pp.1973-1986,2004. 
Breast Cancer Detection by Optimal Classification using GWO Algorithm

[20] Agrawal, P., Vatsa, M., \& Singh, R "Saliency based mass detection from screening mammograms", Signal Processing, vol.99, pp.29-47, 2014.

[21] Seyedali Mirjalili a, Seyed Mohammad Mirjalili, Andrew Lewis, "Grey Wolf Optimizer", Advances in Engineering Software, vol. 69, pp.46-61, 2014.

[22] Yogeswaran Mohan, Sia Seng Chee, Donica Kan Pei Xin and Lee Poh Foong, "Artificial Neural Network for Classification of Depressive and Normal in EEG", 2016 IEEE EMBS Conference on Biomedical Engineering and Sciences (IECBES), 2016.

[23] DHANASHREE GADKARI, "IMAGE QUALITY ANALYSIS USING GLCM", 2004

[24] Leif Kobbelt, "Using the Discrete Fourier Transform to Analyze the Convergence of Subdivision Schemes",Department of Computer Sciences,vol.5, pp. 68-91 May 12, 1997.

[25] YouliYuan, MinZhang, PengfeiLuo, ZabihGhassemlooy, LeiLang, DanshiWang, BoZhang and DahaiHan, " SVMbased detection in visible light communications", Optik, vol.151, pp. 55-64, December 2017.

[26] John McCall, "Genetic algorithms for modelling and optimisation", Journal of Computational and Applied Mathematics, vol. 184, no. 1, pp. 205-222, 1 December 2005.

[27] Junhao Zhang, Pinqi Xia, "An improved PSO algorithm for parameter identification of nonlinear dynamic hysteretic models", Journal of Sound and Vibration, vol. 389, pp. 153-167, 17 February 2017.

[28] Iztok Fister, Iztok Fister, Xin-She Yang, Janez Brest, "A comprehensive review of firefly algorithms", Swarm and Evolutionary Computation, vol. 13, pp. 34-46, December 2013. 